\title{
Incidence and risk factors of posttraumatic seizures following traumatic brain injury: A Traumatic Brain Injury Model Systems Study
}

I,2 Anne C. Ritter, ${ }^{2,3,4,5}$ Amy K. Wagner, 'Anthony Fabio, ${ }^{6,7}$ Mary Jo Pugh, ${ }^{8}$ William C. Walker, ${ }^{9}$ Jerzy P. Szaflarski, ${ }^{10}$ Ross D. Zafonte, ' $A$ Allen W. Brown, ${ }^{12,13}$ Flora M. Hammond, ${ }^{14}$ Tamara Bushnik, ${ }^{15}$ Douglas Johnson-Greene, ${ }^{16}$ Timothy Shea, ${ }^{17}$ Jason W. Krellman, ${ }^{16}$ Joseph A. Rosenthal, and ${ }^{18}$ Laura E. Dreer

Epilepsia, $* *(*): 1-10,2016$

doi: 10.1111/epi.13582

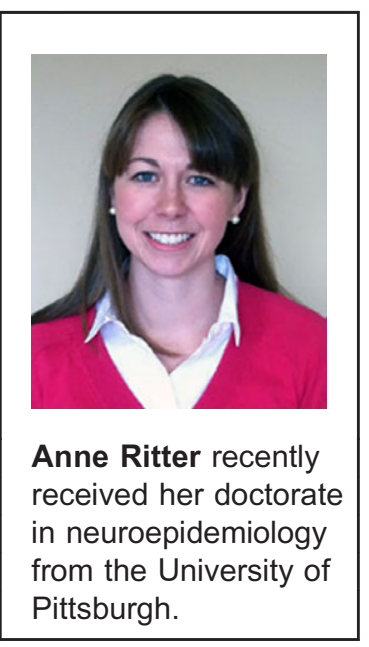

\section{SUMMARY}

Objective: Determine incidence of posttraumatic seizure (PTS) following traumatic brain injury (TBI) among individuals with moderate-to-severe $\mathrm{TBI}$ requiring rehabilitation and surviving at least 5 years.

Methods: Using the prospective TBI Model Systems National Database, we calculated PTS incidence during acute hospitalization, and at years $I, 2$, and 5 postinjury in a continuously followed cohort enrolled from 1989 to $2000(n=795)$. Incidence rates were stratified by risk factors, and adjusted relative risk (RR) was calculated. Late PTS associations with immediate $(<24 \mathrm{~h})$, early $(24 \mathrm{~h}-7$ day), or late seizures ( $>7$ day) versus no seizure prior to discharge from acute hospitalization was also examined.

Results: PTS incidence during acute hospitalization was highest immediately $(<24 \mathrm{~h})$ post-TBI (8.9\%). New onset PTS incidence was greatest between discharge from inpatient rehabilitation and year I (9.2\%). Late PTS cumulative incidence from injury to year I was II.9\%, and reached $20.5 \%$ by year 5 . Immediate/early PTS RR (2.04) was increased for those undergoing surgical evacuation procedures. Late PTS RR was significantly greater for individuals who self-identified as a race other than black/white (year I RR = 2.22), and for black individuals (year $5 R R=3.02$ ) versus white individuals. Late PTS was greater for individuals with subarachnoid hemorrhage (year I $R R=2.06$ ) and individuals age 23-32 (year $5 R R=2.43$ ) and 33-44 (year 5 RR = 3.02). Late PTS RR years I and 5 was significantly higher for those undergoing surgical evacuation procedures (RR: 3.05 and 2.72 , respectively).

Significance: In this prospective, longitudinal, observational study, PTS incidence was similar to that in studies published previously. Individuals with immediate/late seizures during acute hospitalization have increased late PTS risk. Race, intracranial

\footnotetext{
Accepted September 20, 2016

${ }^{1}$ Department of Epidemiology, University of Pittsburgh, Pittsburgh, Pennsylvania, U.S.A.; ${ }^{2}$ Physical Medicine \& Rehabilitation, University of Pittsburgh, Pittsburgh, Pennsylvania, U.S.A.; ${ }^{3}$ Safar Center for Resuscitation Research, University of Pittsburgh, Pittsburgh, Pennsylvania, U.S.A.; ${ }^{4}$ Department of Neuroscience, University of Pittsburgh, Pittsburgh, Pennsylvania, U.S.A.; ${ }^{5}$ Center for Neuroscience at University of Pittsburgh, Pittsburgh, Pennsylvania, U.S.A.; ${ }^{6}$ South Texas Veterans Health Care System Polytrauma Rehabilitation Center, San Antonio, Texas, U.S.A.; ${ }^{7}$ Department of Epidemiology and Biostatistics, University of Texas Health Science Center San Antonio, San Antonio, Texas, U.S.A.; ${ }^{8}$ Department of Physical Medicine \& Rehabilitation, Virginia Commonwealth University, Richmond, Virginia, U.S.A.; ${ }^{9}$ Department of Neurology, UAB Epilepsy Center, University of Alabama at Birmingham, Birmingham, Alabama, U.S.A.; ${ }^{10}$ Spaulding Rehabilitation Hospital, Harvard Medical School, Boston, Massachusetts, U.S.A.; ${ }^{11}$ Department of Physical Medicine and Rehabilitation, Mayo Clinic, Rochester, Minnesota, U.S.A.; ${ }^{12}$ Carolinas Rehabilitation, Charlotte, North Carolina, U.S.A.; ${ }^{13}$ Indiana University School of Medicine, Indianapolis, Indiana, U.S.A.; ${ }^{14}$ Rusk Rehabilitation, New York University School of Medicine, New York, New York, U.S.A.; ${ }^{15}$ Miller School of Medicine, University of Miami, Miami, Florida, U.S.A.; ${ }^{16}$ Department of Physical Medicine and Rehabilitation, Ohio State University, Columbus, Ohio, U.S.A.; ${ }^{17}$ Department of Rehabilitation Medicine, Icahn School of Medicine at Mount Sinai, New York, New York, U.S.A.; and ${ }^{18}$ Departments of Physical Medicine and Rehabilitation and Ophthalmology, The University of Alabama at Birmingham, Birmingham, Alabama, U.S.A.

Address correspondence to Amy K. Wagner, Department of Physical Medicine and Rehabilitation, University of Pittsburgh, 3471 Fifth Avenue Suite 202, Pittsburgh, PA 15213, U.S.A. E-mail: wagnerak@upmc.edu
}

This is the author's manuscript of the article published in final edited form as:

Ritter, A. C., Wagner, A. K., Fabio, A., Pugh, M. J., Walker, W. C., Szaflarski, J. P., ... Dreer, L. E. (2016). Incidence and risk factors of posttraumatic seizures following traumatic brain injury: A Traumatic Brain Injury Model Systems Study. Epilepsia, 57(12), $1968-1977$. https://doi.org/10.1111/epi.13582 
pathologies, and neurosurgical procedures also influenced PTS RR. Further studies are needed to examine the impact of seizure prophylaxis in high-risk subgroups and to delineate contributors to race/age associations on long-term seizure outcomes. KEY WORDS: Epilepsy, Prophylaxis, Relative risk, TBI Model Systems, Epidemiology.

\section{Key Points}

- In this prospective, longitudinal, observational study, PTS incidence was similar to that in previously published studies

- During acute hospitalization, PTS incidence was highest immediately following TBI and associated with late PTS, 1 and 5 years postinjury

- Late PTS first emerging during acute-care hospitalization was associated with PTS at 1 year post-TBI

- Adjusted PTS RR was increased during acute care and years 1 and 5 for those with surgical evacuation

- Adjusted PTS RR was increased for nonwhite individuals and those undergoing surgical evacuation 1 and 5 years post-TBI and by 5 years post-TBI for individuals ages $23-44$ at injury

Traumatic brain injury (TBI) is a prevalent public health problem with an annual incidence of $>2.5$ million in the United States, of which $\sim 12 \%$ results in hospitalization or death. ${ }^{1}$ TBI also has many related secondary chronic conditions $^{2}$ including shorter life expectancy after severe TBI versus the demographically similar, non-TBI populations. ${ }^{3}$ Recent work using the TBI Model Systems (TBIMS) National Database (NDB) found seizure to be an important contributor to premature death among individuals who were hospitalized and received inpatient rehabilitation for TBI. ${ }^{3}$

Posttraumatic seizures (PTS) can occur any time postTBI. Classification is based on the time of seizure postinjury: immediate $(<24 \mathrm{~h})$, early ( $24 \mathrm{~h}-7$ day), or late ( $>7$ day post-TBI). ${ }^{4}$ Cutoffs reflect proposed differences in causal mechanisms and subsequent seizure risk. ${ }^{5,6}$ Seizures occurring within the first week following TBI, also termed acute symptomatic seizures, ${ }^{7}$ are considered transient, decreasing seizure threshold only temporarily. ${ }^{6}$ Late PTS, often used interchangeably with posttraumatic epilepsy (PTE), is characterized by persistent neurobiologic changes, attributed to secondary injury biochemical cascades and epileptogenic mechanisms that eventually present as clinical seizures. ${ }^{8,9}$ Individuals with acute symptomatic seizures who have a subsequent late seizure have late PTS, or PTE. The clinical definition of epilepsy, revised in 2014 by the International League Against Epilepsy (ILAE), includes conditions where an individual has a single unprovoked seizure, and their risk of a recurrent seizure is at least the same risk of seizure recurrence after two unprovoked seizures occurring $\geq 24 \mathrm{~h}$ apart $(\geq 60 \%) .^{6}$ The recurrent seizure risk following a single, unprovoked seizure $>7$ days post-TBI is high enough to consider late PTS as an epileptic condition. ${ }^{6,10}$

Reported PTS incidence varies widely and depends on study design and population characteristics. Few large epidemiologic PTS investigations have been conducted in heterogeneous populations. The seminal population-based study in the United States examined late PTS in a predominantly white population from 1935 to $1984 .{ }^{11}$ Among individuals with severe TBI, the cumulative probability of late PTS was $10.0 \% 5$ years after TBI; early PTS occurred in $2.6 \%$ of individuals. ${ }^{11}$ This study included all ranges of TBI severity, was racially homogenous, and included both adults and children. Including adults and children may confound risk relationships and complicate accurate risk factor determination, since neurologic injury/recovery mechanisms post-TBI may vary over the course of neuro development or aging. These authors also reported $10.3 \%$ of adults with severe TBI developed early PTS. ${ }^{12}$ Other, smaller studies report an early PTS incidence ranging from $2.4 \%$ to $8.4 \% .^{5,13}$ However, these studies also included children and adults with a range of TBI severities. Late PTS cumulative probability rates have been more often reported, and findings vary widely ${ }^{11,14}$; with prevalence ranging from $4 \%$ to

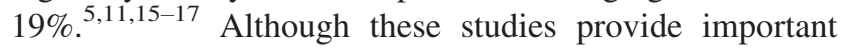
information regarding PTS frequency after TBI, many are retrospective, not racially diverse, from single medical centers, and cannot be generalized to large heterogeneous populations. In addition, they provide little information on immediate and/or early PTS. To address these limitations, the purpose of this study was to prospectively determine PTS incidence following TBI among individuals with moderate-to-severe TBI requiring rehabilitation and surviving at least 5 years using a large-scale, multicenter database.

We used the TBIMS-NDB to calculate multiple PTS frequency measures in a cohort followed out to 5 years postinjury. In addition, we stratified PTS incidence at various time points by demographic and injury characteristics of interest to compute adjusted relative risk (RR) for each factor.

\section{MeThods}

\section{Study design and population}

Data were obtained from the prospective TBIMS-NDB. The TBIMS-NDB is a multicenter, prospective, 
observational study investigating recovery and outcomes following moderate-to-severe TBI and inpatient rehabilitation in a heterogeneous population across the United States. The population followed through the TBIMS-NDB is reported to be representative of individuals with moderateto-severe TBI receiving acute inpatient rehabilitation nationally., ${ }^{3,18}$ All participating sites have an affiliated trauma center with acute neurosurgical capabilities and associated comprehensive inpatient rehabilitation. Eligibility criteria are: moderate-to-severe TBI (posttraumatic amnesia $[\mathrm{PTA}]>24 \mathrm{~h}, \quad$ loss of consciousness $[\mathrm{LOC}]>30 \mathrm{~min}$, or emergency department Glasgow Coma Scale [GCS] score $<13$, or positive neuroimaging findings), age $\geq 16$ years, admitted to a participating hospital emergency department within $24 \mathrm{~h}$ of injury, and receipt of both acute care and inpatient rehabilitation within a TBIMS-designated hospital system. All enrolled individuals, or legal proxy, provided written informed consent; institutional review board approval exists at all sites.

An additional inclusion criterion for this study was completion of year 5 postinjury follow-up interview. Individuals were then excluded if data regarding seizure activity during acute care hospitalization, or year 1 and year 2 postinjury, were not available. The acute hospitalization seizure variable was dropped from TBIMS data collection procedures, and follow-up seizure definitions changed, in 2003 and 2005, respectively. Therefore, all individuals included in analyses were enrolled between 1989 and 2000 across 13 centers; follow-up assessments were completed by 2006.

\section{Data collection}

Data were collected at enrollment, years 1, 2, and 5 postinjury. Enrollment data included demographic, social, and injury characteristics as well as personal and medical history (preinjury), and acute outcomes. A proxy interview was completed for both enrollment and follow-up when participants with TBI were unable to answer questions accurately. Throughout the study duration, data collection protocols changed over time. Therefore, missing data exist even for individuals who completed assessments at all study time points.

\section{Outcome variable}

The main outcome variable was PTS status, determined during the course of acute care hospitalization and at each follow-up time point (years 1, 2, and 5).

\section{PTS during acute care hospitalization}

The presence/absence of a physician-confirmed clinical seizure during acute hospitalization was identified via medical record review using a standardized form following discharge from acute hospitalization and classified based on time from injury (immediate, $<24 \mathrm{~h}$; early, 1-7 days; late, $>7$ days). Only time of first seizure was recorded. Multiple seizures were not captured; therefore, individuals developing seizures immediately or early after injury may have had seizures in a subsequent time category prior to acute discharge.

\section{PTS at follow-up interviews}

At each follow-up, individuals were asked "Have you been told by a physician that you have had a seizure since your last follow-up?" Yes/No answers were recorded on a standardized form; individuals self-reporting seizure activity were documented as having PTS at the specified time point.

\section{PTS risk factors}

Risk factors of interest included demographic and injury characteristics. Demographic variables included age, sex, and race. Injury characteristics included admission Glasgow Coma Scale (GCS) score, pathology on computed tomography (CT) scan obtained within 7 days of injury, penetrating TBI (pTBI), and neurosurgical procedures (Table S1). Injury severity was also classified using alternate criteria for moderate-to-severe injury based on duration of posttraumatic amnesia (PTA), loss of consciousness (LOC), and positive neuroimaging findings. ${ }^{19} \mathrm{CT}$ findings were included as separate variables for specific pathology type (e.g., subdural hematoma [SDH], subarachnoid hemorrhage $[\mathrm{SAH}])$ coded as present/absent, and were not mutually exclusive. pTBI was computed via a coding algorithm validated previously in a subsample of the TBIMS. ${ }^{20} \mathrm{~A}$ contusion load score was calculated by summing the number of regions with contusion on $\mathrm{CT}$ reports, and then collapsing the sum into $0,1-2$, and $\geq 3$ regions. Seizures during acute hospitalization were examined for PTS associations at follow-up (Table 5). No data were collected on premorbid seizure or epilepsy history. International Classification of Diseases, Ninth Revision (ICD-9) codes indicating that neurosurgical procedures or complications were not collected, and neither were medication data.

\section{Statistical analysis}

All statistical analyses were completed using SAS version 9.4 (SAS Institute, Carry, NC, U.S.A.) and R version 3.0.2. ${ }^{21}$ Seizure incidence was calculated during acute care hospitalization and at follow-up. Two-sample independent $t$-tests and chi-square tests were used to determine the differences in population characteristics between the cohort included and not included for analysis (Table S2). At years 1 and 2, if data on seizure activity since last follow-up were missing ( $n=101$ and $n=99$, respectively), seizure status was considered not present at that time point. If individuals had no prior seizure activity, they were considered "at-risk" for PTS and were included in the denominator of incidence calculations. If individuals had evidence of PTS at a previous time point, they were not considered "at-risk" for incident PTS. Sensitivity analyses, including chi-square and Mann-Whitney, or Fisher's exact test and Kruskal-Wallis, 
were conducted to determine whether individuals with missing data at year 1 or year 2 differed from those without missing data. Late PTS incidence since last follow-up was calculated at years 1,2, and 5. Cumulative incidence of late PTS from time of injury was also calculated at each followup time point (i.e., $>7$ days to year $1,>7$ days to year 2 , and so on). The percent of individuals reporting seizure activity at multiple time points was calculated, stratified by time of onset.

Following primary incidence calculations, immediate/ early PTS (injury to 7 days), late PTS from injury to year 1 , and late PTS from year 1 to year 5 incidence rates were stratified by risk factors of interest, and adjusted RRs were reported by applying logistic regression models. We applied the odds ratio, estimated from the logistic model, to approximate RR, since PTS rates were low $(<10 \%) .{ }^{22}$ We also obtained RR estimates using log binomial models. There were no significant differences between OR and RR estimates (data not shown). To assess collinearity between adjusted covariates, variance inflation factors (VIFs) were examined. VIF $\geq 10$ was used to define collinearity. We did not find any collinearity between covariates used to generate adjusted RR. Age was categorized into quartiles, and contusion load was grouped into $0,1-2$, and $\geq 3$. RRs for age, race, sex, and injury severity were derived from mutually adjusted models. RRs for CT-related variables SDH, SAH, intraventricular hemorrhage (IVH), epidural hematoma (EDH), pTBI, and contusion load were adjusted for age, race, sex, and injury severity. Surgical procedures were adjusted for relevant CT-derived injury types and pTBI. For demographic variables identified as having significantly different RRs for PTS, sensitivity analyses were conducted to determine if there were differences in injury characteristics.

Seizures during acute care (immediate, early, and late) were then examined specifically as risk factors. Prior to this analysis, late PTS was recalculated to remove late seizures occurring during acute care ( $>7$ days to discharge) from the case definition. Level-specific (using no acute seizures as the referent group) Fisher exact tests were performed to examine differences in late PTS incidence at years 1 and 5 by acute seizure status.

\section{RESULTS}

\section{Population}

Data were available for 2,418 individuals injured and enrolled prior to 2001. Of these, 796 were assessed at 5 years postinjury and met inclusion criteria (Fig. 1). Individuals included for analysis were significantly more injured, less likely to self-report as white, and were more likely to have intraventricular hemorrhage (Table S2) compared to those not included for analysis. Individuals included for analysis were predominantly men (74.8\%), self-identified as white $(58.3 \%)$, and had a mean age of 35.4 years at injury. The predominant cause of injury was motor vehicle collision
2418 Injured and enrolled prior to 1 Jan 2001

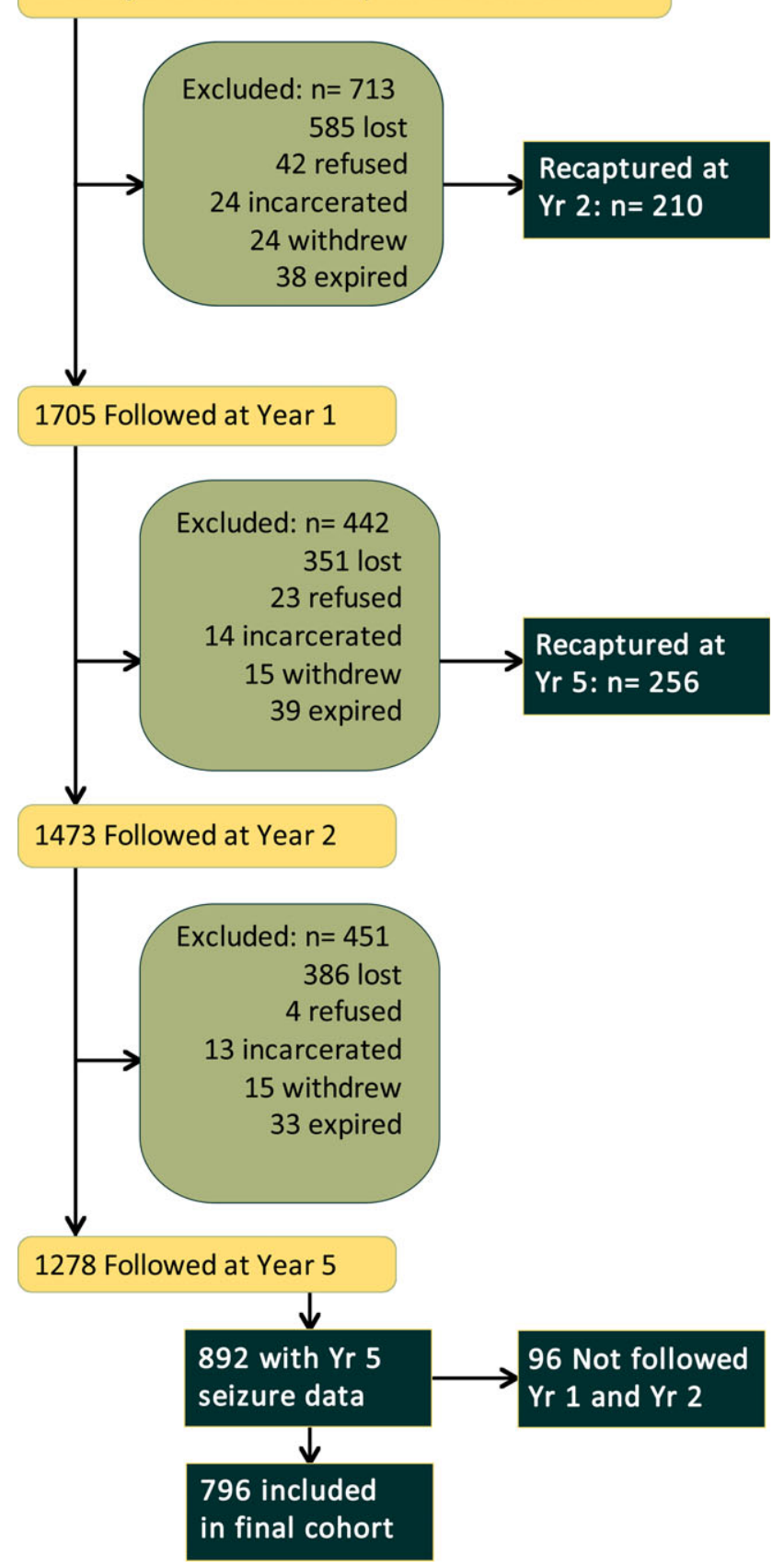

Figure $\mathbf{I}$.

Consolidated Standards of Reporting Trials (CONSORT)-like flow diagram depicting the flow of participants from time of injury to year 5 postinjury.

Epilepsia (C) ILAE

(51.6\%; Table 1). Individuals lost to follow-up at year 1 or 2 who were recaptured at year 5 tended to have less severe injuries and fewer high-risk pathologies (data not shown).

\section{Frequency measures}

By year 5 follow-up, 219 individuals (27.5\%) had reported or documented seizure activity at some point 


\begin{tabular}{|lr|}
\hline \multicolumn{2}{|c|}{ Table I. Demographic and injury characteristics at } \\
baseline visit, N (\%)
\end{tabular}

Table 2. Frequency measures of PTS at follow-up time points after TBI

\begin{tabular}{|c|c|c|c|}
\hline Seizure time point post-TBI & $\begin{array}{c}\text { New-onset } \\
\text { seizure } \\
\text { incidence } \\
\mathrm{N}(\%)\end{array}$ & $\begin{array}{c}\text { Incidence } \\
\text { of late PTS } \\
\text { since last } \\
\text { follow-up } \\
\mathrm{N}(\%)\end{array}$ & $\begin{array}{c}\text { Cumulative } \\
\text { incidence } \\
\text { late PTS } \\
\text { since injury } \\
\mathrm{N}(\%)\end{array}$ \\
\hline Initial population & 796 & 796 & 796 \\
\hline \multicolumn{4}{|l|}{ Acute seizure status } \\
\hline Immediate (<24 h) & 71 (8.9) & - & - \\
\hline Early (I to 7 days) & $14(1.9)$ & - & - \\
\hline Late $(>7$ days $)$ & $13(1.8)$ & - & - \\
\hline Year I & $64(9.2)$ & $86(10.8)$ & $95(11.9)$ \\
\hline Year 2 & $32(5.0)$ & $39(5.5)$ & $134(16.8)$ \\
\hline Year 5 & $25(4.2)$ & $29(4.3)$ & $163(20.5)$ \\
\hline
\end{tabular}

postinjury, and 163 (20.5\%) developed late PTS (Table 2). During acute care hospitalization (mean 22.8 days), 98 individuals $(12.3 \%)$ had seizures, with highest acute hospitalization PTS incidence immediately $(<24 \mathrm{~h})$ after TBI (Table 2); $1.8 \%$ developed incident PTS after 7 days postinjury but prior to acute discharge. Incidence of new onset and late PTS since last follow-up peaked at year 1 (Table 2). Of those developing late PTS by year 5, 58.3\% did so by year 1 , and $82.2 \%$ did so by year 2 . Furthermore, at years 1,2 , and $5,>50 \%$ of individuals reporting seizure activity endorsed multiple seizures since last queried.

Of individuals with first seizure prior to year 5, 38.0\% reported seizure activity at one time interval only. Among
Table 3. Individuals reporting seizures at multiple time points based on time of first seizure

\begin{tabular}{|c|c|c|c|c|}
\hline $\begin{array}{l}\text { Time of first } \\
\text { seizure }(N)\end{array}$ & $\begin{array}{c}\text { Seizure at } \\
\text { I time } \\
\text { point }\end{array}$ & $\begin{array}{c}\text { Seizure at } \\
2 \text { time } \\
\text { points }\end{array}$ & $\begin{array}{c}\text { Seizure at } \\
3 \text { time } \\
\text { points }\end{array}$ & $\begin{array}{c}\text { Seizure } \\
\text { at } 4 \text { time } \\
\text { points }\end{array}$ \\
\hline \multicolumn{5}{|c|}{ Acute seizure status } \\
\hline Immediate (7I) & $44(62.0)$ & $15(2 \mid .1)$ & $9(12.7)$ & $3(4.2)$ \\
\hline Early (14) & $12(85.7)$ & $2(14.3)$ & 0 & 0 \\
\hline Late (I3) & $8(6 \mid .5)$ & I (7.7) & I (7.7) & $2(15.4)$ \\
\hline Year I (64) & $23(35.9)$ & $26(40.6)$ & I5 (23.4) & NA \\
\hline Year 2 (32) & $19(59.9)$ & I $3(40.1)$ & NA & NA \\
\hline Year 5 (25) & $25(100)$ & NA & NA & NA \\
\hline
\end{tabular}

individuals with incident PTS at year 1 or 2, 64\% and $40.1 \%$, respectively, reported interval seizure activity at subsequent follow-ups (Table 3).

\section{Stratified incidence and adjusted relative risks}

Stratified analyses showed PTS incidence was lower among women than men at each time point, but adjusted RRs for sex were not significant. Individuals self-identifying as white also tended to have lower PTS RRs versus individuals not identifying as white, with significant differences evident at years 1 and 5 (Table 4). Further analyses showed differences in pTBI frequencies across race $(2.4 \%$ white, $7.7 \%$ black, $6.3 \%$ other with pTBI $[\mathrm{p}=0.01])$. At 5 years, individuals age 23-44 were more likely to have a seizure versus individuals younger than 22 years of age. Those older than age 45 also had an RR of 1.44, but this result did not reach significance.

PTS incidence was stratified by injury severity measures: moderate versus severe injury as determined by duration of LOC, GCS, PTA, and neuroimaging findings, and no differences in adjusted RR were identified. Immediate/early PTS incidence stratification by pathology showed no patterns. When late PTS incidence was stratified by pathology, only the adjusted RR for SAH pathology and contusion load at year 1 reached significance (Table 4). When evaluated by neurosurgical procedures, adjusted RR was consistently greater (although mostly not significant) for individuals undergoing a procedure versus individuals who did not. However, the adjusted RR was significantly greater for individuals undergoing surgical evacuation of SDH, EDH, or intracerebral hemorrhage at all time points (Table 4).

When late PTS incidence at years 1 and 5 were stratified by time of seizure during acute hospitalization, significant differences were found (Table 5). Individuals with immediate $(\mathrm{p}<0.001)$ and late $(\mathrm{p}=0.028)$ seizures occurring during acute care more frequently developed late PTS between acute discharge and year 1 . Those with immediate seizures also had more frequent seizures at year 5 (Fisher's exact test $\mathrm{p}=0.023$ ). 
A. C. Ritter et al.

Table 4. Incidence and relative risk of PTS stratified by variables of interest during acute care hospitalization

\begin{tabular}{|c|c|c|c|c|c|c|c|c|c|}
\hline & \multicolumn{3}{|c|}{ Immediate and early PTS } & \multicolumn{3}{|c|}{ Late PTS Year I } & \multicolumn{3}{|c|}{ Late PTS Year 5} \\
\hline & \multicolumn{2}{|c|}{$\mathrm{N}(\%)$} & \multirow[b]{2}{*}{$\mathrm{RR}(\mathrm{Cl})$} & \multicolumn{2}{|c|}{$\mathrm{N}(\%)$} & \multirow[b]{2}{*}{$\mathrm{RR}(\mathrm{Cl})$} & \multicolumn{2}{|c|}{ No Seizure } & \multirow[b]{2}{*}{$\mathrm{RR}(\mathrm{Cl})$} \\
\hline & No seizure & Seizure & & No seizure & Seizure & & No seizure & Seizure & \\
\hline Sample size & $7 \mid I(89.3)$ & $85(10.7)$ & & $70 \mathrm{I}(88.1)$ & $95(11.9)$ & & $633(90.3)$ & $68(9.7)$ & \\
\hline \multicolumn{10}{|l|}{$\operatorname{Sex}^{a}$} \\
\hline Men & $53 \mid(89.6)$ & $64(10.8)$ & 1.0 & $519(87.2)$ & $76(12.8)$ & 1.0 & $466(89.8)$ & $53(10.2)$ & 1.0 \\
\hline Women & $180(89.2)$ & $21(10.4)$ & $1.04(0.62-1.76)$ & $182(90.6)$ & $19(9.4)$ & $1.40(0.82-2.38)$ & $167(91.8)$ & $15(8.2)$ & $1.17(0.63-2.17)$ \\
\hline \multicolumn{10}{|l|}{ Race $^{a}$} \\
\hline White & $416(89.7)$ & $48(10.3)$ & 1.0 & $420(90.5)$ & $44(9.5)$ & 1.0 & 395 (94.1) & $25(5.9)$ & 1.0 \\
\hline Black & $218(88.6)$ & $28(11.4)$ & $1.12(0.68-1.85)$ & $211(85.8)$ & $35(14.2)$ & $1.54(0.96-2.48)$ & $175(82.9)$ & $36(17.1)$ & $3.02(1.75-5.22)$ \\
\hline Other & 77 (89.5) & $9(10.5)$ & $1.00(0.47-2.13)$ & $70(81.4)$ & $16(18.6)$ & $2.22(1.18-4.16)$ & $63(90.0)$ & $7(10.0)$ & $1.85(0.76-4.50)$ \\
\hline \multicolumn{10}{|c|}{ Age (quartile) ${ }^{a}$} \\
\hline $16-22$ & $186(89.0)$ & $21(11.0)$ & 1.0 & I $82(87.1)$ & 27 (I2.9) & 1.0 & $173(95.0)$ & $9(5.0)$ & 1.0 \\
\hline $23-32$ & $170(89.0)$ & $21(11.0)$ & $0.98(0.52-1.84)$ & $168(88.0)$ & $23(12.0)$ & $0.90(0.49-1.64)$ & $148(88.1)$ & $20(11.9)$ & $2.43(1.06-5.55)$ \\
\hline $33-44$ & $183(90.2)$ & $20(9.8)$ & $0.87(0.46-1.64)$ & $177(87.2)$ & $26(12.8)$ & $0.96(0.54-1.72)$ & $150(84.8)$ & $27(I 5.2)$ & $3.20(1.45-7.09)$ \\
\hline $45+$ & $172(89.1)$ & $21(10.9)$ & $0.97(0.52-1.83)$ & $174(90.2)$ & $19(9.8)$ & $0.70(0.37-1.31)$ & $162(93.1)$ & $12(6.9)$ & $1.44(0.58-3.54)$ \\
\hline \multicolumn{10}{|c|}{ Injury severity ${ }^{f}$} \\
\hline Moderate & $69(87.3)$ & $10(12.7)$ & 1.0 & $69(87.3)$ & $10(12.7)$ & 1.0 & $63(91.3)$ & $6(8.7)$ & 1.0 \\
\hline Severe & $642(89.5)$ & $75(10.5)$ & $0.80(0.40-1.64)$ & $632(88.2)$ & $85(11.8)$ & $0.87(0.43-1.78)$ & $570(90.2)$ & $62(9.8)$ & I.II (0.45-2.76) \\
\hline \multicolumn{10}{|l|}{$\mathrm{SDH}^{b}$} \\
\hline No & $350(88.4)$ & $46(11.6)$ & 1.0 & $357(90.2)$ & $39(9.8)$ & 1.0 & $332(93.0)$ & $25(7.0)$ & 1.0 \\
\hline Yes & 224 (92.2) & $19(7.8)$ & $0.66(0.37-1.16)$ & $209(86.0)$ & $34(14.0)$ & $1.55(0.94-2.57)$ & $188(90.0)$ & $21(10.0)$ & $1.55(0.82-2.93)$ \\
\hline \multicolumn{10}{|l|}{$\mathrm{SAH}^{b}$} \\
\hline No & $323(89.0)$ & $40(11.0)$ & 1.0 & $333(91.7)$ & $30(8.3)$ & 1.0 & $306(91.9)$ & $27(8.1)$ & 1.0 \\
\hline Yes & $25 \mid(90.9)$ & $25(9.1)$ & $0.81(0.48-1.39)$ & $233(84.4)$ & $43(15.6)$ & $2.06(1.24-3.42)$ & $214(91.9)$ & $19(8.2)$ & $1.04(0.55-1.98)$ \\
\hline \multicolumn{10}{|l|}{$\mathrm{IVH}^{b}$} \\
\hline No & $452(89.2)$ & $55(10.8)$ & 1.0 & $448(88.4)$ & $59(11.6)$ & 1.0 & $4 \mid 3(92.2)$ & $35(7.8)$ & 1.0 \\
\hline Yes & $122(92.4)$ & $10(7.6)$ & $0.68(0.34-1.39)$ & I I 8 (89.4) & $14(10.6)$ & $0.92(0.49-1.72)$ & 107 (90.7) & II (9.3) & I.42 (0.67-2.97) \\
\hline \multicolumn{10}{|l|}{$\mathrm{EDH}^{b}$} \\
\hline No & $503(90.5)$ & $53(9.5)$ & 1.0 & $497(89.4)$ & $59(10.6)$ & 1.0 & $457(92.0)$ & $40(8.1)$ & 1.0 \\
\hline Yes & 71 (85.5) & $12(14.5)$ & $1.62(0.82-3.18)$ & $69(83.1)$ & $14(16.9)$ & $\mathrm{I} .80(0.94-3.4 \mathrm{I})$ & $63(9 \mid .3)$ & $6(8.7)$ & $1.12(0.45-2.8 \mathrm{I})$ \\
\hline \multicolumn{10}{|l|}{$\mathrm{PTBI}^{b}$} \\
\hline No & $53 \mid(89.7)$ & $61(10.3)$ & 1.0 & $525(88.7)$ & $67(11.3)$ & 1.0 & $485(92.4)$ & $40(7.6)$ & 1.0 \\
\hline Yes & $25(92.6)$ & $2(7.4)$ & $0.65(0.15-2.85)$ & $22(8 \mid .5)$ & $5(18.5)$ & I.54 (0.55-4.28) & $18(81.8)$ & $4(18.2)$ & $2.13(0.65-6.95)$ \\
\hline \multicolumn{10}{|c|}{ Contusion load $^{b}$} \\
\hline 0 & $230(88.5)$ & $30(11.5)$ & 1.0 & $238(91.5)$ & $22(8.5)$ & 1.0 & $222(93.3)$ & $16(6.7)$ & 1.0 \\
\hline $\mathrm{I}-2$ & 225 (9I.I) & $22(8.9)$ & $0.76(0.42-1.36)$ & $218(88.3)$ & $29(11.7)$ & $1.40(0.77-2.52)$ & 200 (91.7) & $18(8.3)$ & $1.42(0.69-2.93)$ \\
\hline$\geq 3$ & $119(90.2)$ & $13(9.8)$ & $0.84(0.42-1.68)$ & $110(83.3)$ & $22(16.7)$ & $2.17(1.14-4.12)$ & 98 (89.1) & $12(10.9)$ & $1.75(0.78-3.94)$ \\
\hline $\begin{array}{l}\text { Surgical } \\
\text { evacuation }^{c}\end{array}$ & & & & & & & & & \\
\hline No & $55 \mid(90.9)$ & $55(9.1)$ & 1.0 & $550(90.8)$ & $56(9.2)$ & 1.0 & $510(92.7)$ & $40(7.3)$ & 1.0 \\
\hline Yes & $160(84.2)$ & $30(15.8)$ & $2.04(1.09,3.81)$ & $15 \mid(79.5)$ & $39(20.5)$ & $3.05(1.73,5.37)$ & $123(8 \mid .5)$ & $28(18.5)$ & $2.72(1.28,5.78)$ \\
\hline $\begin{array}{l}\text { Contusion } \\
\text { debridement }\end{array}$ & & & & & & & & & \\
\hline No & $646(89.6)$ & $75(10.4)$ & 1.0 & $642(89.0)$ & 79 (II.) & 1.0 & $583(90.8)$ & $59(9.2)$ & 1.0 \\
\hline Yes & $65(86.7)$ & $10(13.3)$ & $0.85(0.32,2.27)$ & 59 (78.7) & $16(21.3)$ & $\mathrm{I} .34(0.6 \mathrm{I}, 2.97)$ & 50 (84.7) & $9(15.3)$ & I.33 $(0.50,3.52)$ \\
\hline $\begin{array}{l}\text { Depressed sk } \\
\text { fracture } \\
\text { elevation }\end{array}$ & & & & & & & & & \\
\hline No & $663(89.3)$ & $79(10.7)$ & 1.0 & $654(88.3)$ & $87(11.7)$ & 1.0 & $590(90.2)$ & $64(9.8)$ & 1.0 \\
\hline Yes & $49(89.1)$ & $6(10.9)$ & I. $44(0.46,4.49)$ & $47(85.5)$ & $8(14.5)$ & $0.90(0.28,2.87)$ & 43 (91.5) & $4(8.5)$ & I.6I $(0.48,5.46)$ \\
\hline $\begin{array}{l}\text { Removal } \\
\text { skull flap }^{d}\end{array}$ & & & & & & & & & \\
\hline No & $667(89.4)$ & $79(10.6)$ & 1.0 & $662(88.7)$ & $84(11.3)$ & 1.0 & $599(90.5)$ & $63(9.5)$ & 1.0 \\
\hline Yes & $44(88.0)$ & $6(12.0)$ & $1.39(0.56,3.46)$ & $39(78.0)$ & II (22.0) & $2.06(0.95,4.46)$ & $34(87.2)$ & $5(12.8)$ & I.78 $(0.57,5.54)$ \\
\hline
\end{tabular}

SDH, subdural hematoma; SAH, subarachnoid hemorrhage; IVH, intraventricular hemorrhage; EDH, epidural hematoma; pTBI, penetrating TBI; GCS, Glasgow Coma Scale.

${ }^{a}$ Age, race, sex, and injury severity were mutually adjusted.

${ }^{b}$ Adjusted for age, race, sex, and injury severity.

${ }^{\mathrm{C}}$ Adjusted for age, race, sex, injury severity, EDH and SDH, and contusion load.

${ }^{d}$ Adjusted for age, race, sex, injury severity, and contusion load.

${ }^{\mathrm{e}}$ Adjusted for age, race, sex, injury severity, and PTBI.

finjury severity as determined by GCS, duration of loss of consciousness, posttraumatic amnesia, and neuroimaging findings.

Bolded text in this table denote statistically significant $R R$ values 


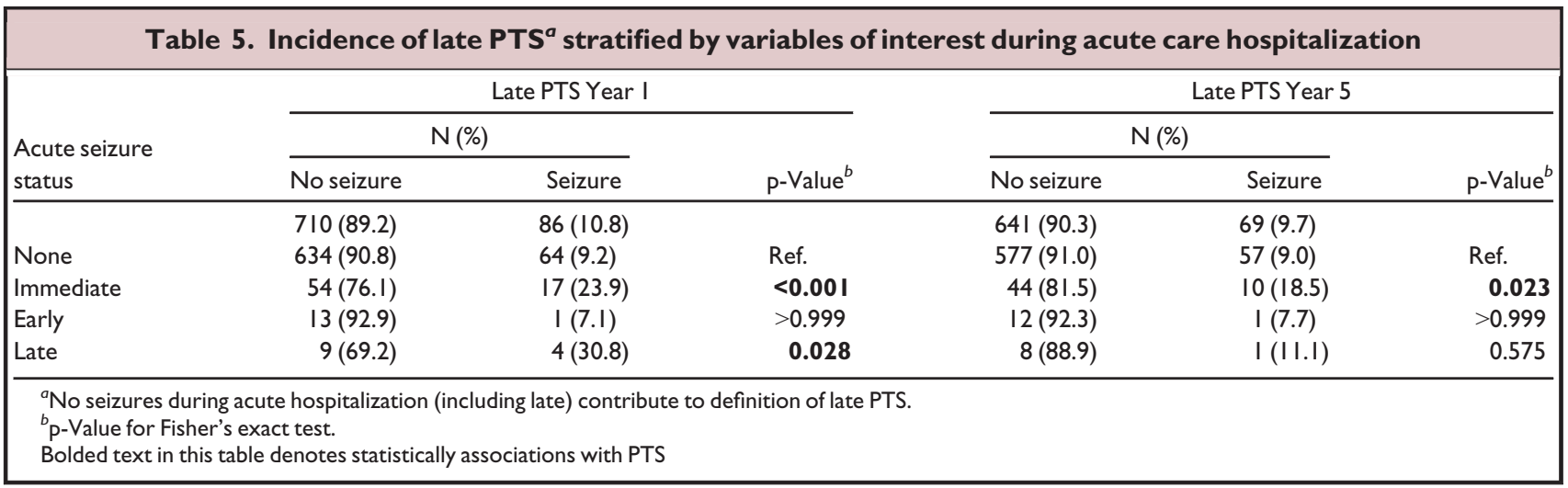

\section{Discussion}

We examined multiple PTS frequency measures and associations with demographic and injury characteristics during and following acute care hospitalization and inpatient rehabilitation for moderate to severe TBI from a population representing a broad cross-section of the moderate to severe TBI cases receiving rehabilitation nationally. ${ }^{18}$ Total PTS incidence during acute hospitalization was slightly higher than previously reported, but early seizure incidence was consistent with previous reports. ${ }^{11,13,23-27}$ However, not all prior PTS studies during acute hospitalization clarify if immediate and early seizures are considered simultaneously. Few previous reports specifically delineate immediate PTS incidence. Annegers and colleagues determined that $2.1 \%$ of a Rochester county Minnesota study cohort, including children and all injury severities injured from 1935 to 1974 , developed early PTS. ${ }^{12}$ Of these, $75.9 \%$ first developed seizures during the initial $24 \mathrm{~h}$ post-TBI, similar to our finding that $72.4 \%$ of individuals who developed PTS during acute hospitalization first developed seizures within $24 \mathrm{~h}$ of injury. These rates are higher than those reported by Jennett, where only $5 \%$ exhibited early seizures with $60 \%$ occurring within 24 h. ${ }^{26}$ Disparate study findings might be due to differences in participants (i.e., injury severity/age) or methods for capturing immediate/early PTS. When considering only adults with severe TBI in Annegers' study, $10.3 \%$ developed early seizures ${ }^{12}$ similar to $10.7 \%$ in our study.

Interval new-onset seizure (9.2\%) and late PTS (10.8\%) incidence were highest at year 1 , similar to previously published findings in comparable study samples. ${ }^{5}$ Annual late PTS incidence since last follow-up at years 1 and $2(10.8 \%$ and $5.5 \%$, respectively) were higher than annual incidence rates reported previously by Ferguson et al. ${ }^{28}$ In our study, the cumulative incidence of late PTS at year 1 (11.9\%) and year $5(20.5 \%)$ were $\geq 2$ times higher than for those with severe TBI in a population-based study of cumulative incidence based on person-time. ${ }^{11}$ In two studies by Englander, using a sample similar to ours, late PTS incidence at 2 years was $16.4 \%$ and $14 \%,{ }^{14,29}$ similar to the $16.8 \%$ in our study.
Differences between the percent affected in our analyses may be attributed to the inclusion of individuals with mild TBI or children. Possible differences between our analysis and that of Annegers could also be attributed to differences in study design (e.g., population-based study with medical chart review versus prospective longitudinal cohort study and self-report). In addition, due to changes in clinical care over time, differences could be attributable to increased electroencephalography (EEG) monitoring of individuals post-TBI, and/or higher survival of the more severely injured in the current study. Future research validating these findings using a more recent cohort from TBIMS-NDB could be considered with reintroduction of seizure data collection. However, due to changes in TBIMS seizure data collection occurring after 2000 , we cannot validate the distinct effects of acute symptomatic seizures or late seizures during acute hospitalization in a more recent, independent TBIMS population.

Consistent with other studies, $82.2 \%$ of individuals developing late PTS did so by year 2 postinjury. ${ }^{10}$ Yet, it is important to recognize new-onset seizures, and subsequently new cases of late PTS, continued to be detected at year 5. Previous PTE studies show new cases may develop as many as 30 years postinjury, and epilepsy risk remains significantly elevated versus the general population up to 10 years postinjury among those with severe TBI. ${ }^{11}$ Our data provide a characterization of late PTS risk out to 5 years post-TBI. Future work should continue to use the TBIMS-NDB to extend the current follow-up time.

Cumulative late PTS incidence reached $20.5 \%$ by year 5 , greater than reported rates of $13.7 \%$ by 5 years postinjury ${ }^{5}$ but lower than rates in combat veteran populations. ${ }^{30}$ Although cohorts from both studies were recruited from rehabilitation facilities, differences in study design and population (e.g., higher pTBI among military populations) likely contributed to differences.

When PTS incidence was stratified by risk variables, patterns in adjusted RR were observed for race, but not sex or injury severity. The lack of significantly greater RRs for men could be confounded by survival bias if men were more severely injured and did not survive to year 5 . However, in a 
A. C. Ritter et al.

more recently injured TBIMS-NDB cohort, men were at higher risk for seizures during acute care hospitalization, even in multivariable models including markers of injury severity. ${ }^{31}$ Although injury severity is a factor reported in other epidemiologic studies describing PTS, ${ }^{23}$ our population included only individuals with moderate-to-severe TBI surviving their injury and qualifying for inpatient rehabilitation, a patient selection bias that may limit the sensitivity of this variable to PTS risk.

Individuals self-identifying as white had a lower risk than nonwhite individuals of late PTS at years 1 and 5. No other known large epidemiologic PTS studies have reported significant differences in risk associated with race. One study demonstrated increased late PTS RR at 2 years for nonwhite groups versus white individuals, but results were not statistically significant. ${ }^{14}$ Racial differences in socioeconomic status may contribute to increased likelihood for injury factors and environmental exposures that may increase PTS risk after TBI (e.g., repeat TBI, substance use). ${ }^{32}$ Differences in PTS frequency by race may also be partially explained by differences in injury type (i.e., pTBI). ${ }^{33}$ In the current population, pTBI prevalence was significantly greater in nonwhites compared to those self-identified as white. However, previous work developing prognostic models for PTS using a more recent cohort from the TBIMS-NDB did not find race to be associated with $\mathrm{PTS}$ and did not investigate race as a PTS predictor in multivariable modeling. ${ }^{31}$ Differences in late PTS risk by race may also potentially reflect genetic differences thought to influence PTS risk. ${ }^{34-37}$ Future work might investigate racial/ethnic differences in epilepsyrelated outcomes and comorbidity burden. ${ }^{38}$

Individuals age 23-44 years had increased incident PTS risk 5 years postinjury. Individuals $>45$ years had an adjusted RR of 1.44 for incident PTS at 5 years, but this finding was not statistically significant, which may be due, in part, to survival bias in this older age group, as follow-up at 5 years was a study inclusion criteria. Increased 5-year PTS incidence for older individuals compared to adolescents/young adults may be varied, but studies suggest that age itself is a risk factor for epilepsy in general. ${ }^{39}$ This risk may reflect a multifactorial cumulative exposure risk from chronic disease factors pertinent to older individuals with TBI, ${ }^{40}$ including cerebrovascular disease, depression, diabetes, alcohol and medication use, CNS injury, and neurodegenerative disease. ${ }^{41}$ Furthermore, evidence suggests TBI pathology reflects many characteristic components observed with neurodegenerative disease (e.g., $\beta$-amyloid deposition $^{42}$ ), and as such, may accelerate brain aging and associated epilepsy risk that accompanies neurodegenerative disease. $^{41}$

Examining incidence stratified by pathology revealed somewhat surprising results. Although incidence patterns were as expected, late PTS risk was only significantly elevated for SAH and higher contusion load at year 1 . These data are inconsistent with most studies, finding that $\mathrm{SDH}$ is a significant PTS risk factor. Our findings may be due to variability within SDH and SAH definitions applied in the TBIMS-NDB over time. Of note, at years 1 and 5, pTBI was associated with the highest late PTS frequencies of any characteristic studied (18.5\% and $18.2 \%$, respectively). Differences in RR were not statistically significant, but low pTBI numbers suggest that these analyses were underpowered.

Examining adjusted RRs from neurosurgical procedures showed results consistent with existing literature, wherein neurosurgical procedures increase risk of subsequent seizure. ${ }^{11,14,15,28,43,44}$ Adjusted RR at all PTS time points was significantly greater for individuals undergoing surgical evacuation of hemorrhage and was greatest at year 1 (RR 3.00 , 95\% confidence interval [CI] 1.72-5.24). Adjusted RRs were nonsignificant for other procedures, which may be due to the relatively low numbers of individuals with PTS also having these procedures. In addition, an invasive procedure like skull-flap removal (i.e. craniectomy) may be underpowered to detect significant differences due to small sample size, likely attributable to this cohort's time of injury (1989-2000), when craniectomy was not as common a procedure as today. These procedure variable findings are limited by data collection procedures, which denote only that the specified procedure was completed, and not if the procedure was repeated. In addition, procedures were not mutually exclusive, and a single participant may have undergone conservative intervention prior to progressing to more invasive procedures as medically necessary. When generating RR results, we accounted for potential confounding by adjusting for demographics, injury severity, and CT/injury type as outlined in the results. However, other sources of residual confounding likely exist that were not captured by the covariates available for analysis.

When late PTS incidence at years 1 and 5 was recalculated, not including individuals with first seizure occurring late ( $>7$ day post-TBI) during acute hospitalization in the case definition, frequencies were significantly different based on timing of first acute seizure. Individuals with immediate seizure $(<24 \mathrm{~h})$ had a higher late PTS incidence and greater risk at both time points versus those without seizures during acute hospitalization. Those with late seizures ( $>7$ days) during acute hospitalization also had a higher late PTS incidence at year 1 versus individuals without seizures acutely (Table 5). In our analyses, immediate/early seizures were mutually exclusive due to limitations in data collection; therefore, we were unable to examine the impact of having both immediate and early seizures on late PTS risk.

The high percentage of individuals in our study with immediate seizures, and relationship of immediate seizure to seizures at years 1 and 5 post-TBI, is a novel finding. Historically, immediate seizures are considered "provoked," and as such, are not considered a contributor to PTE risk or epileptogenesis after TBI. Yet our data showing an association with late seizures at 1 and 5 years suggest immediate 
seizures may influence epileptogenesis and PTE risk after TBI. We hypothesize that immediate seizure activity can overactivate excitatory amino acid (EAA) receptors, ${ }^{8}$ thereby perpetuating TBI-induced excitotoxicity and contributing to epileptogenesis. ${ }^{45}$ Because there are no proven treatments to prevent seizures and affect epileptogenesis, future work might consider how targeted prevention and treatment of immediate seizures might influence PTE risk.

Although these data provide PTS characterization in a large cohort of individuals from the United States, limitations must be considered. Our cohort is restricted to individuals having received inpatient rehabilitation and surviving to year 5 post-TBI. In addition, PTS/PTE definitions and data collection methods used can bias descriptive analyses. We cannot specify the data source used as evidence of seizure during acute hospitalization other than to cite "documentation from the medical record." It is unclear if evidence was collected using EEG. Currently, continuous EEG (cEEG) is common in many trauma centers, but how cEEG might have factored into acute symptomatic seizure identification for individuals injured prior to 2001 is less certain. In critically ill patients with TBI, up to $50 \%$ of seizures are reported to be subclinical. ${ }^{46}$ In addition, only the first seizure during acute care hospitalization was documented. Therefore, individuals with immediate or early seizures, and having later seizures during acute hospitalization, were not accounted for in late PTS calculations if they did not experience a seizure after discharge from acute hospitalization. Similarly, subsequent seizures during acute care hospitalization, if occurring, were not documented.

Another potential limitation is PTS misclassification at follow-up because, while acute symptomatic seizure information was obtained via medical record, all follow-up data were based on participant or proxy self-report of whether a physician told them they had a seizure. Individuals could overreport if they experienced psychogenic nonepileptic seizures or transient neurologic symptoms not due to seizure activity. Conversely, individuals experiencing true epileptic seizure activity between follow-ups, who were not aware or did not seek care, may contribute to underestimating late PTS. Nevertheless, in large epidemiologic studies of seizure and epilepsy, self-report remains common and necessary. ${ }^{47}$ Adding to possible misclassification bias, individuals with missing data at years 1 and 2 were included as at-risk individuals for incidence calculations. By doing so, we may have included individuals as "atrisk" who developed seizures, underestimating the true incidence. However, we examined individuals with missing PTS data in our cohort, and similar to other studies where less severely injured individuals have lower followup rates, ${ }^{48,49}$ we determined they typically had less severe injury and less intracranial pathology identified via CT than those contacted for all follow-ups (data not shown). Within the larger TBIMS-NDB, individuals with less severe TBI who presumably recover better, are more often lost to follow-up than individuals with more complex injuries and presumably worse outcomes. ${ }^{50}$ Therefore, excluding individuals with missing data at year 1 or year 2 , who were subsequently followed at year 5, would have likely produced a greater degree of bias and overestimated PTS incidence. There were significant differences (race, injury severity, and IVH) between those included versus not included in the analysis, suggesting some potential limitations with the external validity of the study in addition to external validity limitations that may accompany the selection bias of TBI-MS participation. Due to limitations in data collection, we were unable to identify, and subsequently exclude, individuals with preexisting epilepsy disorders. In addition, those with previous neurologic injury (e.g., previous stroke and concussion) are not excluded from the TBIMS-NDB, further confounding the possibility of attributing PTS solely to the index TBI. Despite these limitations, the current analysis suggests age, race, CT findings, and neurosurgical procedures as possible PTS risk factors. The work provides insight into temporal PTS risk factors, including acute hospital seizure, and examines the effect of each on late PTS incidence out to 5 years postinjury.

\section{ACKNOWLEDgements}

The National Institute on Disability, Independent Living, and Rehabilitation Research (NIDILRR) supported the collection of original data for this manuscript. The contents of this manuscript were developed under grants NIDILRR Grants 90DP0041 (AKW, ACR), NIDILRR 90DP0038 (JWK), 90DP0036 (FH), 90DP0033 (WCW), and the Polytrauma Rehabilitation Center Traumatic Brain Injury Model System (MJP). The Polytrauma Rehabilitation Center TBI Model System is a funded collaboration between the Department of Veterans Affairs (VA) and NIDILRR. NIDILRR is a Center within the Administration for Community Living (ACL), Department of Health and Human Services (HHS). The contents of this manuscript do not necessarily represent the policy of the VA, NIDILRR, ACL, or HHS, and endorsement of this content by the Federal Government should not be assumed.

\section{Disclosure}

No author has any relevant conflict of interest. We confirm we have read the Journal's position on issues involved in ethical publication and affirm this report is consistent with those guidelines

\section{REFERENCES}

1. Report to Congress on Traumatic Brain Injury in the United States: Epidemiology and Rehabilitation. Atlanta, GA: Centers for Disease Control and Prevention; 2014.

2. Corrigan JD, Hammond FM. Traumatic brain injury as a chronic health condition. Arch Phys Med Rehabil 2013;94:1199-1201.

3. Harrison-Felix C, Pretz C, Hammond FM, et al. Life expectancy after inpatient rehabilitation for traumatic brain injury in the United States. $J$ Neurotrauma 2015;32:1893-1901.

4. Practice parameter: antiepileptic drug treatment of posttraumatic seizures. Brain Injury Special Interest Group of the American Academy of Physical Medicine and Rehabilitation. Arch Phys Med Rehabil 1998;79:594-597. 
5. Asikainen I, Kaste M, Sarna S. Early and late posttraumatic seizures in traumatic brain injury rehabilitation patients: brain injury factors causing late seizures and influence of seizures on long-term outcome. Epilepsia 1999;40:584-589.

6. Fisher RS, Acevedo C, Arzimanoglou A, et al. ILAE official report: a practical clinical definition of epilepsy. Epilepsia 2014;55:475-482.

7. Beghi E, Carpio A, Forsgren L, et al. Recommendation for a definition of acute symptomatic seizure. Epilepsia 2010;51:671-675.

8. Agrawal A, Timothy J, Pandit L, et al. Post-traumatic epilepsy: an overview. Clin Neurol Neurosurg 2006;108:433-439.

9. Hunt RF, Boychuk JA, Smith BN. Neural circuit mechanisms of posttraumatic epilepsy. Front Cell Neurosci 2013;7:89.

10. Haltiner AM, Temkin NR, Dikmen SS. Risk of seizure recurrence after the first late posttraumatic seizure. Arch Phys Med Rehabil 1997;78:835-840.

11. Annegers JF, Hauser WA, Coan SP, et al. A population-based study of seizures after traumatic brain injuries. N Engl J Med 1998;338:20-24.

12. Annegers JF, Grabow JD, Groover RV, et al. Seizures after head trauma: a population study. Neurology 1980;30:683-689.

13. Lee ST, Lui TN. Early seizures after mild closed head injury. J Neurosurg 1992;76:435-439.

14. Englander J, Bushnik T, Duong TT, et al. Analyzing risk factors for late posttraumatic seizures: a prospective, multicenter investigation. Arch Phys Med Rehabil 2003;84:365-373.

15. Angeleri F, Majkowski J, Cacchio G, et al. Posttraumatic epilepsy risk factors: one-year prospective study after head injury. Epilepsia 1999;40:1222-1230.

16. Jennett WB, Lewin W. Traumatic epilepsy after closed head injuries. $J$ Neurol Neurosurg Psychiatry 1960;23:295-301.

17. Zhao Y, Wu H, Wang X, et al. Clinical epidemiology of posttraumatic epilepsy in a group of Chinese patients. Seizure 2012;21:322-326.

18. Pretz CR, Cuthbert JP, Whiteneck GG. A validation study for using iterative proportional fitting to weight the Traumatic Brain Injury Model Systems National Database: an NIDRR-sponsored study. Arch Phys Med Rehabil 2015;96:746-749.

19. Brasure M, Lamberty GJ, Sayer NA, et al. Multidisciplinary postacute rehabilitation for moderate to severe traumatic brain injury in adults. Rockville, MD: Comparative Effectiveness Review No. 72. Agency for Healthcare Research and Quality; 2012.

20. Walker WC, Ketchum JS 3rd, Marwitz JH, et al. Global outcome and late seizures after penetrating versus closed traumatic brain injury: a NIDRR TBI Model Systems Study. J Head Trauma Rehabil 2015;30:231-240.

21. R Foundation for Statistical Computing. $R$ : a language and environment for statistical computing. [computer program]. Vienna, Austria: R Foundation for Statistical Computing; 2013.

22. Bonita R, Beaglehole R, Kjellstrom T. Basic epidemiology. 2nd Ed. Geneva: World Health Organization; 2006.

23. Frey LC. Epidemiology of posttraumatic epilepsy: a critical review. Epilepsia 2003;44(Suppl. 10):11-17.

24. Najafi MR, Tabesh H, Hosseini H, et al. Early and late posttraumatic seizures following traumatic brain injury: a five-year follow-up survival study. Adv Biomed Res 2015;4:82.

25. Desai BT, Whitman S, Coonley-Hoganson R, et al. Seizures in relation to head injury. Ann Emerg Med 1983;12:543-546.

26. Jennett B. Early traumatic epilepsy. Incidence and significance after nonmissile injuries. Arch Neurol 1974;30:394-398.

27. Oluwole OS. Incidence and risk factors of early post-traumatic seizures in Nigerians. Brain Inj 2011;25:980-988.

28. Ferguson PL, Smith GM, Wannamaker BB, et al. A population-based study of risk of epilepsy after hospitalization for traumatic brain injury. Epilepsia 2010;51:891-898.

29. Englander J, Bushnik T, Wright JM, et al. Mortality in late post-traumatic seizures. J Neurotrauma 2009;26:1471-1477.

30. Raymont V, Salazar AM, Lipsky R, et al. Correlates of posttraumatic epilepsy 35 years following combat brain injury. Neurology 2010;75:224-229.

31. Ritter AC, Wagner AK, Szaflarski JP, et al. Prognostic models for predicting post-traumatic seizures during acute hospitalization and at 1 and 2 years following traumatic brain injury. Epilepsia 2016;57:1503-1514.

32. Saunders LL, Selassie AW, Hill EG, et al. A population-based study of repetitive traumatic brain injury among persons with traumatic brain injury. Brain Inj 2009;23:866-872.
33. Wagner AK, Sasser HC, Hammond FM, et al. Intentional traumatic brain injury: epidemiology, risk factors, and associations with injury severity and mortality. J Trauma 2000;49:404-410.

34. Diamond ML, Ritter AC, Jackson EK, et al. Genetic variation in the adenosine regulatory cycle is associated with posttraumatic epilepsy development. Epilepsia 2015;56:1198-1206.

35. Diamond ML, Ritter AC, Failla MD, et al. IL-1beta associations with posttraumatic epilepsy development: a genetics and biomarker cohort study. Epilepsia 2014;55:1109-1119.

36. Wagner AK, Miller MA, Scanlon J, et al. Adenosine A1 receptor gene variants associated with post-traumatic seizures after severe TBI. Epilepsy Res 2010;90:259-272.

37. Ritter AC, Kammerer CM, Brooks MM, et al. Genetic variation in neuronal glutamate transporters accelerates epileptogenesis and increases risk for post-traumatic seizures. Epilepsia 2016;57:984-993.

38. Szaflarski M, Szaflarski JP, Privitera MD, et al. Racial/ethnic disparities in the treatment of epilepsy: what do we know? What do we need to know? Epilepsy Behav 2006;9:243-264.

39. Hauser WA, Annegers JF, Rocca WA. Descriptive epidemiology of epilepsy: contributions of population-based studies from Rochester, Minnesota. Mayo Clin Proc 1996;71:576-586.

40. Kumar RG, Juengst SB, Wang Z. Epidemiology of comorbid conditions among older adults with traumatic brain injury. J Head Trauma Rehabil (in press 2016).

41. Liu S, Yu W, Lu Y. The causes of new-onset epilepsy and seizures in the elderly. Neuropsychiatr Dis Treat 2016;12:1425-1434.

42. Hong YT, Veenith T, Dewar D, et al. Amyloid imaging with carbon 11-labeled Pittsburgh compound B for traumatic brain injury. JAMA Neurol 2014;71:23-31.

43. Andriessen TM, Horn J, Franschman G, et al. Epidemiology, severity classification, and outcome of moderate and severe traumatic brain injury: a prospective multicenter study. J Neurotrauma 2011;28:20192031.

44. Temkin NR. Risk factors for posttraumatic seizures in adults. Epilepsia 2003;44(Suppl. 10):18-20.

45. Andriessen TM, Jacobs B, Vos PE. Clinical characteristics and pathophysiological mechanisms of focal and diffuse traumatic brain injury. $J$ Cell Mol Med 2010;14:2381-2392.

46. Vespa PM, Nuwer MR, Nenov V, et al. Increased incidence and impact of nonconvulsive and convulsive seizures after traumatic brain injury as detected by continuous electroencephalographic monitoring. J Neurosurg 1999;91:750-760.

47. Fisher RS, Blum DE, DiVentura B, et al. Seizure diaries for clinical research and practice: limitations and future prospects. Epilepsy Behav 2012;24:304-310

48. Corrigan JD, Harrison-Felix C, Bogner J, et al. Systematic bias in traumatic brain injury outcome studies because of loss to follow-up. Arch Phys Med Rehabil 2003;84:153-160.

49. Langley J, Johnson S, Slatyer M, et al. Issues of loss to follow-up in a population study of traumatic brain injury (TBI) followed to 3 years post-trauma. Brain Inj 2010;24:939-947.

50. Krellman JW, Kolakowsky-Hayner SA, Spielman L, et al. Predictors of follow-up completeness in longitudinal research on traumatic brain injury: findings from the National Institute on Disability and Rehabilitation Research traumatic brain injury model systems program. Arch Phys Med Rehabil 2014;95:633-641.

\section{SUPPORTING INFORMATION}

Additional Supporting Information may be found in the online version of this article:

Table S1. Risk factors for stratified incidence and relative risk calculations.

Table S2. Comparison of population characteristics between individuals included in current analysis and individuals not included based on inclusion criteria, $\mathrm{N}(\%)$. 